\title{
Deriving the system equations of unbalanced two-phase induction motor
}

\author{
Hany Ibrahim Shousha, Abdelsamie B. Kotb
}

Department of Electrical Engineering, Al-Azhar University, Egypt

\begin{tabular}{|c|c|}
\hline Article Info & ABSTRACT \\
\hline Article history: & \multirow{6}{*}{$\begin{array}{l}\text { As there is no system driven especially for the two-phase induction motor fed } \\
\text { from unbalanced two-phase supply yet, so we start for derivation the system } \\
\text { equations for the said motor to be generally used even for the balanced or } \\
\text { unbalanced two-phase supply. In this paper, we will derive a system equation } \\
\text { starting from the sequence equivalent circuit for the forward and backwards } \\
\text { equivalent circuits, then we will re-arrange the equations with some } \\
\text { mathematical assumptions which will lead us to the new system equations. } \\
\text { first for the voltage equations then for the current equations and finally for } \\
\text { both power and torque equations. Moreover, we will put an example which } \\
\text { will cover all cases with specific values and relations charts. }\end{array}$} \\
\hline Received Aug 20, 2019 & \\
\hline Revised Oct 06, 2019 & \\
\hline Accepted Feb 18, 2020 & \\
\hline Keywords: & \\
\hline $\begin{array}{l}\text { Forward and } \\
\text { equivalent circuits }\end{array}$ & \\
\hline
\end{tabular}

Performance of two-phase motor under unbalanced supply Sequence circuits

This is an open access article under the $\underline{C C B Y-S A}$ license.

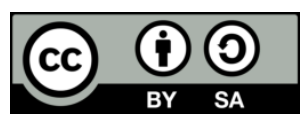

\section{Corresponding Author:}

Hany Ibrahim Shousha,

Department of Electrical Engineering, Al-Azhar University, Egypt.

Email: h.i.shousha@gmail.com

\section{INTRODUCTION}

When the motor works under a general operating conditions of unbalanced stator voltages or windings, and if we start to derive the system equations, it is necessary to start from the known equivalent circuits which are obtained for both forward and back word components [1]. Using a special analysis which will be required in order to completely describe the two-phase equivalent circuit or the system of the voltage equations for the two-phase motor [2]. Using the relations between the sequence and phase values, to obtain the important system of voltage equations for the case of unbalanced system [3]. After the derivation of the system equations we can learn the performance of the unbalanced two-phase electrical motor, using the symmetrical components representation for both voltages and currents [4]. These system equations will be used for all cases for the two-phase induction motor which fed from two-phase power supply under all conditions [5].

\section{RESEARCH METHOD}

\subsection{Equivalent circuits of forward and backward components}

From the usual equivalent circuits of forward and backward components, the following equations for both components are shown in Figure 1. From the figure, we can express the voltage equations as the following:

$$
\begin{aligned}
V_{s f} & =\left(R_{s}+j x_{L S}+j x_{m}\right) I_{s f}+j x_{m} I_{r f} \\
V_{r f} & =J x_{m} I_{s f}+\left(j x_{m}+R_{r} / s\right) I_{r f} \\
V_{s b} & =\left(R_{s}+j x_{L S}+j x_{m}\right) I_{s b}+j x_{m} I_{r b} \\
V_{r b} & =j x_{m} I_{s b}+\left(j x_{m}+R_{r} /(2-s)\right) I_{r b}
\end{aligned}
$$




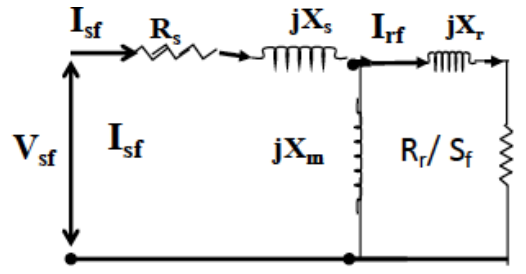

(a)

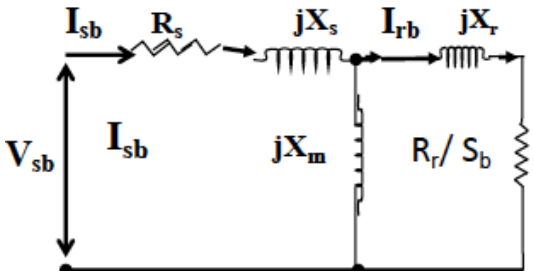

(b)

Figure 1. Single-phase equivalent circuits for a two-phase motor under unbalanced condition (a) forward field and (b) backward field

For the above equivalent circuits there are some assumptions

$$
\begin{array}{ll}
Z_{\mathrm{m}}=\mathrm{j} X_{\mathrm{m}} & \mathrm{Z}_{\mathrm{s}}=\mathrm{R}_{\mathrm{s}}+\mathrm{j} X \mathrm{~s} ., \\
\mathrm{Z}_{\mathrm{sm}}=\mathrm{Z}_{\mathrm{m}}+\mathrm{Z}_{\mathrm{s}} & \mathrm{Z}_{\mathrm{mr}}=\mathrm{Z}_{\mathrm{m}}+\mathrm{j} \mathrm{X}_{\mathrm{r}}+\left(\mathrm{R}_{\mathrm{r}} / \mathrm{S}_{\mathrm{f}}\right) \\
\mathrm{S}_{\mathrm{f}}=\mathrm{S}, & \mathrm{S}_{\mathrm{b}}=(2-\mathrm{S})
\end{array}
$$

The symmetrical components relations for the voltages are

$$
\begin{aligned}
& V_{\mathrm{sf}}=\left(\mathrm{V}_{\mathrm{s} 1}+\mathrm{j} \mathrm{V}_{\mathrm{s} 2}\right) / 2, \mathrm{~V}_{\mathrm{sb}}=\left(\mathrm{V}_{\mathrm{s} 1}-\mathrm{j} \mathrm{V}_{\mathrm{s} 2}\right) / 2, \\
& \mathrm{~V}_{\mathrm{rf}}=\left(\mathrm{V}_{\mathrm{r} 1}+\mathrm{j} \mathrm{V}_{\mathrm{r} 2}\right) / 2, \mathrm{~V}_{\mathrm{rb}}=\left(\mathrm{V}_{\mathrm{r} 1}-\mathrm{j} \mathrm{V}_{\mathrm{r} 2}\right) / 2
\end{aligned}
$$

and for the currents are

$$
\begin{array}{ll}
I_{\mathrm{sf}}=\left(\mathrm{I}_{\mathrm{s} 1}+\mathrm{j} \mathrm{I}_{\mathrm{s} 2}\right) / 2, & \mathrm{I}_{\mathrm{sb}}=\left(\mathrm{I}_{\mathrm{s} 1}-\mathrm{j} \mathrm{I}_{\mathrm{s} 2}\right) / 2, \\
\mathrm{I}_{\mathrm{rf}}=\left(\mathrm{I}_{\mathrm{r} 1}+\mathrm{j} \mathrm{I}_{\mathrm{r} 2}\right) / 2, & \mathrm{I}_{\mathrm{rb}}=\left(\mathrm{I}_{\mathrm{r} 1}-\mathrm{j} \mathrm{I}_{\mathrm{r} 2}\right) / 2 .
\end{array}
$$

\subsection{Solution of the forward and backward currents}

Mathematical analysis for derivation of the system equations is to obtain the system equations of the voltages and currents.

\subsubsection{For voltage system equations using the symmetrical components}

For voltage system equations using the symmetrical components, we use:

$$
\begin{array}{ll}
\mathrm{V}_{\mathrm{s} 1}=\left(\mathrm{V}_{\mathrm{sf}}+\mathrm{V}_{\mathrm{sb}}\right), & \mathrm{V}_{\mathrm{s} 2}=-\mathrm{j}\left(\mathrm{V}_{\mathrm{sf}}-\mathrm{V}_{\mathrm{sb}}\right), \\
\mathrm{V}_{\mathrm{r} 1}=\left(\mathrm{V}_{\mathrm{rf}}+\mathrm{V}_{\mathrm{rb}}\right), & \mathrm{V}_{\mathrm{r} 2}=-\mathrm{j}\left(\mathrm{V}_{\mathrm{rf}}-\mathrm{V}_{\mathrm{rb}}\right),
\end{array}
$$

The similar relations for the currents are:

$$
\begin{array}{ll}
I_{s 1}=\left(I_{s f}+I_{s b}\right), & I_{s 2}=-j\left(I_{s f}-I_{s b}\right), \\
I_{r 1}=\left(I_{r f}+I_{r b}\right), & I_{r 2}=-j\left(I_{r f}-I_{r b}\right),
\end{array}
$$

From the previous analysis, the above system of equations, can be used to obtain the following general system of equations for the phase voltages of the two-phase induction machine in the general forms.

$$
\begin{aligned}
V_{s 1} & =\left(V_{s f}+V_{s b}\right) \\
& =\left(R_{s}+j x_{L S}+j x_{m}\right) I_{s f}+j x_{m} I_{r f}+\left(R_{s}+j x_{L S}+j x_{m}\right) I_{s b}+j x_{m} I_{r b} \\
& =\left(R_{s}+j x_{L S}+j x_{m}\right) \cdot\left(I_{s f}+I_{s b}\right)+j x_{m}\left(I_{r f}+I_{r b}\right)
\end{aligned}
$$

using the previous current equations we get

$$
V_{s 1}=\left(R_{s}+j x_{L S}+j x_{m}\right) \cdot I_{s 1}+j x_{m} \cdot I_{r 1}
$$

By the same manner,

$$
\begin{aligned}
\mathrm{V}_{\mathrm{s} 2} & =-\mathrm{j}\left(\mathrm{V}_{\mathrm{sf}}-\mathrm{V}_{\mathrm{sb}}\right) \\
& =-\mathrm{j}\left(\left(\mathrm{R}_{\mathrm{s}}+\mathrm{j} \mathrm{x}_{\mathrm{LS}}+\mathrm{j} \mathrm{x}_{\mathrm{m}}\right) \mathrm{I}_{\mathrm{sf}}+\mathrm{j} \mathrm{x}_{\mathrm{m}} \mathrm{I}_{\mathrm{rf}}-\left(\mathrm{R}_{\mathrm{s}}+\mathrm{j} \mathrm{x}_{\mathrm{LS}}+\mathrm{j} \mathrm{x}_{\mathrm{m}}\right) \mathrm{I}_{\mathrm{sb}}-\mathrm{j} \mathrm{x}_{\mathrm{m}} \mathrm{I}_{\mathrm{rb}}\right. \\
& =\left(\left(\left(\mathrm{R}_{\mathrm{s}}+\mathrm{j} \mathrm{x}_{\mathrm{LS}}+\mathrm{j} \mathrm{x}_{\mathrm{m}}\right) .-\mathrm{j}\left(\mathrm{I}_{\mathrm{sf}}-\mathrm{I}_{\mathrm{sb}}\right)+\mathrm{j} \mathrm{x}_{\mathrm{m}}\left(-\mathrm{j}\left(\mathrm{I}_{\mathrm{rf}}-\mathrm{I}_{\mathrm{rb}}\right)\right)\right.\right. \\
\mathrm{V}_{\mathrm{s} 2} & =\left(\mathrm{R}_{\mathrm{s}}+\mathrm{j} \mathrm{x}_{\mathrm{LS}}+\mathrm{j} \mathrm{x}_{\mathrm{m}}\right) . \mathrm{I}_{\mathrm{s} 2}+\mathrm{j} \mathrm{x}_{\mathrm{m}} \mathrm{I}_{\mathrm{r} 2}
\end{aligned}
$$


For the other voltage equations

$$
\begin{aligned}
& V_{r 1}=\left(V_{r f}+V_{r b}\right) \\
& V_{r f}=J x_{m} I_{s f}+\left(j x_{m}+R_{r} / s\right) I_{r f} \\
& V_{r b}=j x_{m} I_{s b}+\left(j x_{m}+\left(R_{r} /(2-s)\right)\right) I_{r b} \\
& V_{r 1}=j x_{m} I_{s f}+\left(j x_{m}+R_{r} / s\right) I_{r f}+j x_{m} I_{s b}+\left(j x_{m}+\left(R_{r} /(2-s)\right)\right) I_{r b}
\end{aligned}
$$

We will divide the previous equation into two parts to solve them separately then we will collect them again:

$$
V_{r 1}=\left[j x_{m} I_{s f}+j x_{m} I_{s b .}\right]+\left[\left(j x_{m}+R_{r} / s\right) I_{r f}+\left(j x_{m}+\left(R_{r} /(2-s)\right)\right) I_{r b}\right]
$$

For the first part

$$
\left[j x_{m} I_{s f}+j x_{m} I_{s b .}\right]=j x_{m}\left(I_{s f}+I_{s b}\right)=j x_{m} I_{s 1}
$$

For the seconsd part

$$
\begin{aligned}
& {\left[\left(\mathrm{j} \mathrm{x}_{\mathrm{m}}+\mathrm{R}_{\mathrm{r}} / \mathrm{s}\right) \mathrm{I}_{\mathrm{rf}}+\left(\mathrm{j} \mathrm{x}_{\mathrm{m}}+\left(\mathrm{R}_{\mathrm{r}} /(2-\mathrm{s})\right)\right) \mathrm{I}_{\mathrm{rb}}\right]=} \\
& =\mathrm{j} \mathrm{x}_{\mathrm{m}}\left(\mathrm{I}_{\mathrm{rf}}+\underline{\mathrm{I}}_{\mathrm{rb}}\right)+\left(\mathrm{R}_{\mathrm{r}} / \mathrm{s}\right) \mathrm{I}_{\mathrm{rf}}+\left(\mathrm{R}_{\mathrm{r}} /(2-\mathrm{s})\right) \mathrm{I}_{\mathrm{rb}} \\
& =\mathrm{j} \mathrm{x}_{\mathrm{m}} \mathrm{I}_{\mathrm{r} 1}+\left((2-\mathrm{s}) \mathrm{R}_{\mathrm{r}} /(\mathrm{s}(2-\mathrm{s}))\right) \mathrm{I}_{\mathrm{rf}}+\left(\mathrm{sR}_{\mathrm{r}} /(\mathrm{s}(2-\mathrm{s}))\right) \mathrm{I}_{\mathrm{rb}} \\
& =\mathrm{jx}_{\mathrm{m}} \mathrm{I}_{\mathrm{r} 1}+\left[\left(\mathrm{R}_{\mathrm{r}} /(\mathrm{s}(2-\mathrm{s}))\right) \cdot\left((2-\mathrm{s}) \mathrm{I}_{\mathrm{rf}}+\mathrm{s} \mathrm{I}_{\mathrm{rb}}\right)\right] \\
& =\mathrm{j} \mathrm{x}_{\mathrm{m}} \mathrm{I}_{\mathrm{r} 1}+\left(\mathrm{R}_{\mathrm{r}} /(\mathrm{s}(2-\mathrm{s}))\right) \cdot\left[(1+1-\mathrm{s}) \mathrm{I}_{\mathrm{rf}}-(-1+1-\mathrm{s}) \mathrm{I}_{\mathrm{rb}}\right] \\
& =j x_{m} I_{r 1}+\left(R_{r} /(s(2-s))\right) \cdot\left[I_{r f}+I_{r b}+(1-s) \cdot\left(I_{r f}-I_{r b}\right)\right] \\
& =\mathrm{jx}_{\mathrm{m}} \mathrm{I}_{\mathrm{r} 1}+\left(\mathrm{R}_{\mathrm{r}} /(\mathrm{s}(2-\mathrm{s}))\right) \cdot\left[\mathrm{I}_{\mathrm{r} 1}+\mathrm{j}(1-\mathrm{s}) \mathrm{I}_{\mathrm{r} 2}\right] \\
& =\left[j x_{m} I_{r 1}+\left(R_{r} /(s(2-s))\right)\right] I_{r 1}+j(1-s)\left[\left(R_{r} /(s(2-s))\right) \cdot I_{r 2}\right.
\end{aligned}
$$

Then for both parts

$$
\mathrm{V}_{\mathrm{r} 1}=\mathrm{j} \mathrm{x}_{\mathrm{m}} \mathrm{I}_{\mathrm{s} 1}+\left[\mathrm{j} \mathrm{x}_{\mathrm{m}}+\left(\mathrm{R}_{\mathrm{r}} /(\mathrm{s}(2-\mathrm{s}))\right)\right] \mathrm{I}_{\mathrm{r} 1}+\mathrm{j}(1-\mathrm{s})\left[\left(\mathrm{R}_{\mathrm{r}} /(\mathrm{s}(2-\mathrm{s}))\right) . \mathrm{I}_{\mathrm{r} 2}\right.
$$

for the following equation

$$
\begin{aligned}
& V_{r 2}=-j\left(V_{r f}-V_{r b}\right), \\
& V_{r f}=j x_{m} I_{s f}+\left(j x_{m}+R_{r} / s\right) I_{r f}, \quad V_{r b}=j x_{m} I_{s b}+\left(j x_{m}+\left(R_{r} /(2-s)\right)\right) I_{r b}
\end{aligned}
$$

as the same for the previous equation of the $\mathrm{V}_{\mathrm{r} 1}$

$$
\begin{aligned}
\mathrm{V}_{\mathrm{r} 2} & =-j\left[j x_{m} I_{s f}+\left(j x_{m}+R_{r} / s\right) I_{r f}-j x_{m} I_{s b}-\left(j x_{m}+\left(R_{r} /(2-s)\right)\right) I_{r b}\right. \\
& =-j\left[j x_{m} .\left(I_{s f}-I_{s b}\right)+j x_{m} .\left(I_{r f}-I_{r b}\right)+R_{r} / s . I_{r f}-\left(R_{r} /(2-s)\right) I_{r b}\right. \\
& =-j\left[\left(-x_{m} . I_{s 2}\right)-\left(x_{m} \cdot I_{r 2}\right)+\left[R_{r} / s . I_{r f}-\left(R_{r} /(2-s)\right) I_{r b}\right]\right]
\end{aligned}
$$

for the second part of the equation

$$
\begin{aligned}
\mathrm{R}_{\mathrm{r}} / \mathrm{s} & \left.. \mathrm{I}_{\mathrm{rf}}-\left(\mathrm{R}_{\mathrm{r}} /(2-\mathrm{s})\right) \mathrm{I}_{\mathrm{rb}}\right] \\
& =\left((2-\mathrm{s}) \mathrm{R}_{\mathrm{r}} /(\mathrm{s}(2-\mathrm{s}))\right) \mathrm{I}_{\mathrm{rf}}-\left(\mathrm{sR}_{\mathrm{r}} /(\mathrm{s}(2-\mathrm{s}))\right) \mathrm{I}_{\mathrm{rb}} \\
& =\left(\mathrm{R}_{\mathrm{r}} /(\mathrm{s}(2-\mathrm{s}))\right) \cdot\left((2-\mathrm{s}) \mathrm{I}_{\mathrm{rf}}-\mathrm{s}_{\mathrm{rb}}\right) \\
& =\left(\mathrm{R}_{\mathrm{r}} /(\mathrm{s}(2-\mathrm{s}))\right) \cdot\left[(1+1-\mathrm{s}) \mathrm{I}_{\mathrm{rf}}+(-1+1-\mathrm{s}) \mathrm{I}_{\mathrm{rb}}\right] \\
& =\left(\mathrm{R}_{\mathrm{r}} /(\mathrm{s}(2-\mathrm{s}))\right) \cdot\left[\mathrm{j}(-\mathrm{j})\left(\mathrm{I}_{\mathrm{rf}}-\mathrm{I}_{\mathrm{rb}}\right)+(1-\mathrm{s}) \cdot\left(\mathrm{I}_{\mathrm{rf}}+\mathrm{I}_{\mathrm{rb}}\right)\right] \\
& =\left(\mathrm{R}_{\mathrm{r}} /(\mathrm{s}(2-\mathrm{s}))\right)\left[\mathrm{j} \mathrm{I}_{\mathrm{r} 2}+\mathrm{j}(1-\mathrm{s}) \cdot \mathrm{I}_{\mathrm{r} 1}\right]
\end{aligned}
$$

Then for both parts

$$
\begin{aligned}
\mathrm{j} \mathrm{V}_{\mathrm{r} 2}= & {\left[(1-\mathrm{s}) \cdot \mathrm{R}_{\mathrm{r}} /(\mathrm{s}(2-\mathrm{s}))\right) \cdot \mathrm{I}_{\mathrm{r} 1}+\left(\mathrm{R}_{\mathrm{r}} /(\mathrm{s}(2-\mathrm{s}))+\mathrm{j} \mathrm{x}_{\mathrm{m}}\right) \cdot \mathrm{I}_{\mathrm{r} 1} } \\
& +\mathrm{j}\left(\mathrm{R}_{\mathrm{r}} \cdot(1-\mathrm{s}) /(\mathrm{s}(2-\mathrm{s}))\right) \cdot \mathrm{I}_{\mathrm{r} 2}
\end{aligned}
$$

Then we drive the following system equations for voltages

$$
\begin{aligned}
V_{s 1}= & \left(R_{s}+j x_{L S}+j x_{m}\right) \cdot I_{s 1}+j x_{m} \cdot I_{r 1} \\
j V_{s 2}= & \left(R_{s}+j x_{L S}+j x_{m}\right) \cdot j I_{s 2}+j x_{m} j I_{r 2} \\
V_{r 1}= & j x_{m} I_{s 1}+\left[j x_{m}+\left(R_{r} /(s(2-s))\right)\right] I_{r 1}+j(1-s)\left[\left(R_{r} /(s(2-s))\right) . I_{r 2}\right. \\
j V_{r 2}= & {\left[(1-s) \cdot R_{r} /(s(2-s))\right) \cdot I_{r 1}+\left(R_{r} /(s(2-s))+j x_{m}\right) . I_{r 1} } \\
& +j\left(R_{r} \cdot(1-s) /(s(2-s))\right) \cdot I_{r 2}
\end{aligned}
$$




\subsubsection{For current system equations}

Using the previous equations for the single-phase equivalent circuits will give as the following:

$$
\begin{aligned}
& \mathrm{V}_{\mathrm{sf}}=\mathrm{Z}_{\mathrm{sm}} \cdot \mathrm{I}_{\mathrm{sf}}-\mathrm{Z}_{\mathrm{m}} \cdot \mathrm{I}_{\mathrm{rf}} \\
& \mathrm{V}_{\mathrm{rf}}=\mathrm{Z}_{\mathrm{mr}} \cdot \mathrm{I}_{\mathrm{rf}}-\mathrm{Z}_{\mathrm{m}} \cdot \mathrm{I}_{\mathrm{sf}} \\
& \mathrm{I}_{\mathrm{rf}}=\left(\mathrm{Z}_{\mathrm{sm}} \cdot \mathrm{I}_{\mathrm{sf}}\right) / \mathrm{Z}_{\mathrm{m}}-\mathrm{V}_{\mathrm{sf}} / \mathrm{Z}_{\mathrm{m}}
\end{aligned}
$$

Also, according to the relation between current, impedance and voltage

$$
\mathrm{I}_{\mathrm{rf}}=\left(\mathrm{Z}_{\mathrm{m}} / \mathrm{Z}_{\mathrm{mr}}\right) \cdot \mathrm{I}_{\mathrm{sf}}
$$

Then,

$$
\begin{aligned}
& \mathrm{I}_{\mathrm{rf}}=\left(\mathrm{Z}_{\mathrm{m}} / \mathrm{Z}_{\mathrm{mr}}\right) \cdot \mathrm{I}_{\mathrm{sf}}=\left(\mathrm{Z}_{\mathrm{sm}} \cdot \mathrm{I}_{\mathrm{sf}}\right) / \mathrm{Z}_{\mathrm{m}}-\mathrm{V}_{\mathrm{sf}} / \mathrm{Z}_{\mathrm{m}} \\
& \mathrm{V}_{\mathrm{sf}} / \mathrm{Z}_{\mathrm{m}}=\left[\left(\mathrm{Zsm}_{\mathrm{m}} / \mathrm{Z}_{\mathrm{m}}\right)-\left(\mathrm{Z}_{\mathrm{m}} / \mathrm{Z}_{\mathrm{mr}}\right)\right] \cdot \mathrm{I}_{\mathrm{sf}} \\
& \mathrm{I}_{\mathrm{sf}}=\left[\mathrm{Z}_{\mathrm{mr}} /\left(\left(\mathrm{Z}_{\mathrm{sm}} \cdot \mathrm{Z}_{\mathrm{mr}}\right)-\mathrm{Z}_{\mathrm{m}}{ }^{2}\right)\right] \cdot \mathrm{V}_{\mathrm{sf}}
\end{aligned}
$$

Now we get the first current equation as following

$$
\begin{aligned}
& \mathrm{I}_{\mathrm{sf}}=\left[\mathrm{Z}_{\mathrm{mr}} /\left(\left(\mathrm{Z}_{\mathrm{sm}} \cdot \mathrm{Z}_{\mathrm{mr}}\right)-\mathrm{Z}_{\mathrm{m}}{ }^{2}\right)\right] \cdot \mathrm{V}_{\mathrm{sf}} \\
& \mathrm{I}_{\mathrm{sf}}=\left[\left(\mathrm{Z}_{\mathrm{m}}+\mathrm{j} \mathrm{X}_{\mathrm{r}}+\left(\mathrm{R}_{\mathrm{r}} / \mathrm{S}_{\mathrm{f}}\right)\right) /\left(\left(\left(\mathrm{Z}_{\mathrm{m}}+\mathrm{Z}_{\mathrm{s}}\right) \cdot\left(\mathrm{Z}_{\mathrm{m}}+\mathrm{j} \mathrm{X}_{\mathrm{r}}+\left(\mathrm{R}_{\mathrm{r}} / \mathrm{S}_{\mathrm{f}}\right)\right)\right)-\mathrm{Z}_{\mathrm{m}}{ }^{2}\right)\right] \cdot \mathrm{V}_{\mathrm{sf}}
\end{aligned}
$$

Using the following equation

$$
\mathrm{V}_{\mathrm{rf}}=\mathrm{Z}_{\mathrm{mr}} \cdot \mathrm{I}_{\mathrm{rf}}-\mathrm{Z}_{\mathrm{m}} \cdot \mathrm{I}_{\mathrm{sf}}=0
$$

Then we get

$$
\begin{aligned}
& \mathrm{I}_{\mathrm{rf}}=\left[\mathrm{Z}_{\mathrm{m}} /\left(\left(\mathrm{Z}_{\mathrm{sm}} \cdot \mathrm{Z}_{\mathrm{mr}}\right)-\mathrm{Z}_{\mathrm{m}}{ }^{2}\right)\right] \cdot \mathrm{V}_{\mathrm{sf}} \\
& \mathrm{I}_{\mathrm{rf}}=\left[\mathrm{Z}_{\mathrm{m}} /\left(\left(\left(\mathrm{Z}_{\mathrm{m}}+\mathrm{Z}_{\mathrm{s}}\right) \cdot\left(\mathrm{Z}_{\mathrm{m}}+\mathrm{j} \mathrm{X}_{\mathrm{r}}+\left(\mathrm{R}_{\mathrm{r}} / \mathrm{S}_{\mathrm{f}}\right)\right)\right)-\mathrm{Z}_{\mathrm{m}}{ }^{2}\right)\right] \cdot \mathrm{V}_{\mathrm{sf}}
\end{aligned}
$$

Then we can express for the forward as the following

$$
\begin{aligned}
& \mathrm{I}_{\mathrm{sf}}=\left[\left(\mathrm{Z}_{\mathrm{m}}+\mathrm{j} \mathrm{X}_{\mathrm{r}}+\left(\mathrm{R}_{\mathrm{r}} / \mathrm{S}_{\mathrm{f}}\right)\right) /\left(\left(\left(\mathrm{Z}_{\mathrm{m}}+\mathrm{Z}_{\mathrm{s}}\right) \cdot\left(\mathrm{Z}_{\mathrm{m}}+\mathrm{j} \mathrm{X}_{\mathrm{r}}+\left(\mathrm{R}_{\mathrm{r}} / \mathrm{S}_{\mathrm{f}}\right)\right)\right)-\mathrm{Z}_{\mathrm{m}}^{2}\right)\right] \cdot \mathrm{V}_{\mathrm{sf}} \\
& \mathrm{I}_{\mathrm{rf}}=\left[\mathrm{Z}_{\mathrm{m}} /\left(\left(\left(\mathrm{Z}_{\mathrm{m}}+\mathrm{Z}_{\mathrm{s}}\right) \cdot\left(\mathrm{Z}_{\mathrm{m}}+\mathrm{j} \mathrm{X}_{\mathrm{r}}+\left(\mathrm{R}_{\mathrm{r}} / \mathrm{S}_{\mathrm{f}}\right)\right)\right)-\mathrm{Z}_{\mathrm{m}}{ }^{2}\right)\right] \cdot \mathrm{V}_{\mathrm{sf}}
\end{aligned}
$$

For the backwards,

$$
\begin{aligned}
& \mathrm{I}_{\mathrm{sb}}=\left[\left(\mathrm{Z}_{\mathrm{m}}+\mathrm{j} \mathrm{X}_{\mathrm{r}}+\left(\mathrm{R}_{\mathrm{r}} / \mathrm{S}_{\mathrm{b}}\right)\right) /\left(\left(\left(\mathrm{Z}_{\mathrm{m}}+\mathrm{Z}_{\mathrm{s}}\right) \cdot\left(\mathrm{Z}_{\mathrm{m}}+\mathrm{j} \mathrm{X}_{\mathrm{r}}+\left(\mathrm{R}_{\mathrm{r}} / \mathrm{S}_{\mathrm{b}}\right)\right)\right)-\mathrm{Z}_{\mathrm{m}}^{2}\right)\right] \cdot \mathrm{V}_{\mathrm{sb}} \\
& \mathrm{I}_{\mathrm{rb}}=\left[\mathrm{Z}_{\mathrm{m}} /\left(\left(\left(\mathrm{Z}_{\mathrm{m}}+\mathrm{Z}_{\mathrm{s}}\right) \cdot\left(\mathrm{Z}_{\mathrm{m}}+\mathrm{j} \mathrm{X}_{\mathrm{r}}+\left(\mathrm{R}_{\mathrm{r}} / \mathrm{S}_{\mathrm{b}}\right)\right)\right)-\mathrm{Z}_{\mathrm{m}}{ }^{2}\right)\right] \cdot \mathrm{V}_{\mathrm{sb}}
\end{aligned}
$$

To obtain the air gab power equation

$$
I_{r f}=\left(I_{r 1}+j I_{r 2}\right) / 2, \quad I_{r b}=\left(I_{r 1}-j I_{r 2}\right) / 2
$$

For the $\mathrm{P}_{\mathrm{gf}}$ we use the expression

$$
\begin{aligned}
\mathrm{P}_{\mathrm{gf}} & =\mathrm{m}_{\mathrm{s}} \mathrm{I}_{\mathrm{rf}} \mathrm{I}_{\mathrm{rf}}{ }^{*} \mathrm{R}_{\mathrm{r}} / \mathrm{s}_{\mathrm{f}}, \\
2 \mathrm{I}_{\mathrm{rf}} & =\mathrm{I}_{\mathrm{r} 1 \mathrm{r}}+\mathrm{j} \mathrm{I}_{\mathrm{r} 1 \mathrm{i}}+\mathrm{j} \mathrm{I}_{\mathrm{r} 2 \mathrm{r}}-\mathrm{I}_{\mathrm{r} 2 \mathrm{i}} \\
& =\left(\mathrm{I}_{\mathrm{r} 1 \mathrm{r}}-\mathrm{I}_{\mathrm{r} 2 \mathrm{i}}\right)+\mathrm{j}\left(\mathrm{I}_{\mathrm{r} 1 \mathrm{i}}+\mathrm{I}_{\mathrm{r} 2 \mathrm{r}}\right)
\end{aligned}
$$

Then

$$
\begin{aligned}
2 I_{r f} & =\left(I_{r 1 r}-I_{r 2 i}\right)+j\left(I_{r 1 i}+I_{r 2 r}\right), 2 I_{r f}^{*}=\left(I_{r 1 r}-I_{r 2 i}\right)-j\left(I_{r 1 i}+I_{r 2 r}\right) \\
P_{g f} & =m_{s} I_{r f} I_{r f}^{*} R_{r} / s_{f} \\
& =\left[( ( m _ { s } \cdot R _ { r } ) / 4 s ) \cdot \left(\left(\left(I_{r 1 r}-I_{r 2 i}\right)+j\left(I_{r 1 i}+I_{r 2 r}\right)\right) \cdot\left(\left(I_{r 1 r}-I_{r 2 i}\right)-j\left(I_{r 1 i}+I_{r 2 r}\right)\right)\right.\right. \\
& =\left[\left(\left(m_{s} \cdot R_{r}\right) / 4 s\right) \cdot\left(\left(I_{r 1 r}-I_{r 2 i}\right)^{2}+\left(I_{r 1 i}+I_{r 2 r}\right)^{2}\right)\right. \\
& \left.=\left[\left(\left(m_{s} \cdot R_{r}\right) / 4 s\right) \cdot\left(I_{r 1 r}^{2}-2 I_{r 1 r} \cdot I_{r 2 i}+I_{r 2 i}^{2}+I_{r 1 i}{ }^{2}+2 I_{r 1 i} \cdot I_{r 2 r}+I_{r 2 r}^{2}\right)\right)\right]
\end{aligned}
$$

And for, $\mathrm{I}_{\mathrm{rb}}$

$$
\begin{aligned}
& \mathrm{P}_{\mathrm{gb}}=\mathrm{m}_{\mathrm{s}} \mathrm{I}_{\mathrm{rb}} \mathrm{I}_{\mathrm{rb}}{ }^{*} \mathrm{R}_{\mathrm{r}} / \mathrm{s}_{\mathrm{b}}, \\
& \mathrm{I}_{\mathrm{rb}}=\left(\mathrm{I}_{\mathrm{r} 1}-\mathrm{j} \mathrm{I}_{\mathrm{r} 2}\right) / 2 .
\end{aligned}
$$


With the same manner

then:

$$
\begin{aligned}
2 \mathrm{I}_{\mathrm{rb}} & =\mathrm{I}_{\mathrm{r} 1 \mathrm{r}}+\mathrm{j} \mathrm{I}_{\mathrm{r} 1 \mathrm{i}}-\mathrm{j} \mathrm{I}_{\mathrm{r} 2 \mathrm{r}}+\mathrm{I}_{\mathrm{r} 2 \mathrm{i}} \\
& =\left(\mathrm{I}_{\mathrm{r} 1 \mathrm{r}}+\mathrm{I}_{\mathrm{r} 2 \mathrm{i}}\right)+\mathrm{j}\left(\mathrm{I}_{\mathrm{r} 1 \mathrm{i}}-\mathrm{I}_{\mathrm{r} 2 \mathrm{r}}\right)
\end{aligned}
$$

$$
\begin{aligned}
2 \mathrm{I}_{\mathrm{rb}} & =\left(\mathrm{I}_{\mathrm{r} 1 \mathrm{r}}+\mathrm{I}_{\mathrm{r} 2 \mathrm{i}}\right)+\mathrm{j}\left(\mathrm{I}_{\mathrm{r} 1 \mathrm{i}}-\mathrm{I}_{\mathrm{r} 2 \mathrm{r}}\right), \quad 2 \mathrm{I}_{\mathrm{rb}}{ }^{*}=\left(\mathrm{I}_{\mathrm{r} 1 \mathrm{r}}+\mathrm{I}_{\mathrm{r} 2 \mathrm{i}}\right)-\mathrm{j}\left(\mathrm{I}_{\mathrm{r} 1 \mathrm{i}}-\mathrm{I}_{\mathrm{r} 2 \mathrm{r}}\right) \\
\mathrm{P}_{\mathrm{gb}} & =\mathrm{m}_{\mathrm{s}} \mathrm{I}_{\mathrm{rb}} \mathrm{I}_{\mathrm{rb}}{ }^{*} \mathrm{R}_{\mathrm{r}} / \mathrm{s}_{\mathrm{b}}, \\
& =\left[\left(\left(\mathrm{m}_{\mathrm{s}} \cdot \mathrm{R}_{\mathrm{r}}\right) / 4 \mathrm{~s}_{\mathrm{b}}\right) \cdot\left(\left(\mathrm{I}_{\mathrm{r} 1 \mathrm{r}}+\mathrm{I}_{\mathrm{r} 2 \mathrm{i}}\right)+\mathrm{j}\left(\mathrm{I}_{\mathrm{r} 1 \mathrm{i}}-\mathrm{I}_{\mathrm{r} 2 \mathrm{r}}\right)\right) \cdot\left(\left(\mathrm{I}_{\mathrm{r} 1 \mathrm{r}}+\mathrm{I}_{\mathrm{r} 2 \mathrm{i}}\right)-\mathrm{j}\left(\mathrm{I}_{\mathrm{r} 1 \mathrm{i}}-\mathrm{I}_{\mathrm{r} 2 \mathrm{r}}\right)\right)\right. \\
& =\left[( ( \mathrm { m } _ { \mathrm { s } } \cdot \mathrm { R } _ { \mathrm { r } } ) / 4 \mathrm { s } _ { \mathrm { b } } ) \cdot \left(\left(\mathrm{I}_{\mathrm{r} 1 \mathrm{r}}+\mathrm{I}_{\mathrm{r} 2 \mathrm{i}}\right)^{2}+\left(\left(\mathrm{I}_{\mathrm{r} 1 \mathrm{i}}-\mathrm{I}_{\mathrm{r} 2 \mathrm{r}}\right)^{2}\right)\right.\right. \\
& \left.=\left[\left(\left(\mathrm{m}_{\mathrm{s}} \cdot \mathrm{R}_{\mathrm{r}}\right) / 4 \mathrm{~s}_{\mathrm{b}}\right) \cdot\left(\mathrm{I}_{\mathrm{r} 1 \mathrm{r}}^{2}+2 \mathrm{I}_{\mathrm{r} 1 \mathrm{r}} \cdot \mathrm{I}_{\mathrm{r} 2 \mathrm{i}}+\mathrm{I}_{\mathrm{r} 2 \mathrm{i}}{ }^{2}+\mathrm{I}_{\mathrm{r} 1 \mathrm{i}}{ }^{2}-2 \mathrm{I}_{\mathrm{r} 1 \mathrm{i}} \cdot \mathrm{I}_{\mathrm{r} 2 \mathrm{r}}+\mathrm{I}_{\mathrm{r} 2 \mathrm{r}}{ }^{2}\right)\right)\right]
\end{aligned}
$$

where

$$
\begin{aligned}
\mathrm{P}_{\mathrm{g}}= & \mathrm{P}_{\mathrm{gf}}-\mathrm{P}_{\mathrm{gb}} \\
= & {\left.\left[\left(\left(\mathrm{m}_{\mathrm{s}} \cdot \mathrm{R}_{\mathrm{r}}\right) / 4 \mathrm{~s}\right) \cdot\left(\mathrm{I}_{\mathrm{r} 1 \mathrm{r}}^{2}-2 \mathrm{I}_{\mathrm{r} 1 \mathrm{r}} \cdot \mathrm{I}_{\mathrm{r} 2 \mathrm{i}}+\mathrm{I}_{\mathrm{r} 2 \mathrm{i}}{ }^{2}+\mathrm{I}_{\mathrm{r} 1 \mathrm{i}}{ }^{2}+2 \mathrm{I}_{\mathrm{r} 1 \mathrm{i}} \cdot \mathrm{I}_{\mathrm{r} 2 \mathrm{r}}+\mathrm{I}_{\mathrm{r} 2 \mathrm{r}}{ }^{2}\right)\right)\right]- } \\
& {\left.\left[\left(\left(\mathrm{m}_{\mathrm{s}} \cdot \mathrm{R}_{\mathrm{r}}\right) / 4 \mathrm{~s}_{\mathrm{b}}\right) \cdot\left(\mathrm{I}_{\mathrm{r} 1 \mathrm{r}}{ }^{2}+2 \mathrm{I}_{\mathrm{r} 1 \mathrm{r}} \cdot \mathrm{I}_{\mathrm{r} 2 \mathrm{i}}+\mathrm{I}_{\mathrm{r} 2 \mathrm{i}}{ }^{2}+\mathrm{I}_{\mathrm{r} 1 \mathrm{i}}{ }^{2}-2 \mathrm{I}_{\mathrm{r} 1 \mathrm{i}} \cdot \mathrm{I}_{\mathrm{r} 2 \mathrm{r}}+\mathrm{I}_{\mathrm{r} 2 \mathrm{r}}{ }^{2}\right)\right)\right] } \\
= & {\left[\left(\left(\mathrm{m}_{\mathrm{s}} \cdot \mathrm{R}_{\mathrm{r}}\right) /(2 \mathrm{~s} \cdot(2-\mathrm{s}))\right) \cdot\left(\mathrm{I}_{\mathrm{r} 1 \mathrm{r}}{ }^{2}+\mathrm{I}_{\mathrm{r} 2 \mathrm{i}}{ }^{2}+\mathrm{I}_{\mathrm{r} 2 \mathrm{r}}{ }^{2}+(1-\mathrm{s}) \cdot\left(2 \mathrm{I}_{\mathrm{r} 1 \mathrm{r}} \cdot \mathrm{I}_{\mathrm{r} 2 \mathrm{i}}+2 \mathrm{I}_{\mathrm{r} 1 \mathrm{i}} \cdot \mathrm{I}_{\mathrm{r} 2 \mathrm{r}}+\mathrm{I}_{\mathrm{rli}}{ }^{2}\right)\right)\right.}
\end{aligned}
$$

For the power equations

$$
\begin{aligned}
& \mathrm{P}_{\mathrm{gf}}=\mathrm{m}_{\mathrm{s}} \mathrm{I}_{\mathrm{rf}} \mathrm{I}_{\mathrm{rf}}{ }^{*} \mathrm{R}_{\mathrm{r}} / \mathrm{s}_{\mathrm{f}}, \\
& \mathrm{P}_{\mathrm{gb}}=\mathrm{m}_{\mathrm{s}} \mathrm{I}_{\mathrm{rb}} \mathrm{I}_{\mathrm{rb}}{ }^{*} \mathrm{R}_{\mathrm{r}} / \mathrm{s}_{\mathrm{b}},
\end{aligned}
$$

where, $s_{\mathrm{f}}=\mathrm{s}$ and $\mathrm{s}_{\mathrm{b}}=2-\mathrm{s}$

$$
\begin{aligned}
\mathrm{P}_{\mathrm{g}} & =\mathrm{P}_{\mathrm{gf}}-\mathrm{P}_{\mathrm{gb}} \\
\mathrm{Pm} & =\mathrm{Pg} \cdot(1-\mathrm{s}) \\
\mathrm{T} & =\mathrm{Pg} / \omega_{\mathrm{s}}
\end{aligned}
$$

where $\omega s=\omega / p$, with $p=$ number of pole pair.

\subsection{Performance of two-phase motor under unbalanced supply}

Case study for different values of the phase angle of the second voltage source uses Matlab software for simulation the results according to different values of the voltage angle values $\left(90^{\circ} 60^{\circ} 30^{\circ} 15^{\circ} 0^{\circ}\right)$ and other assumptions as the following

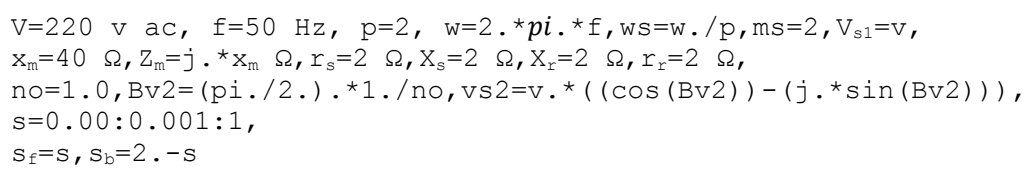

From the previous values and using Matlab we can get the result as in Figures 2-5.

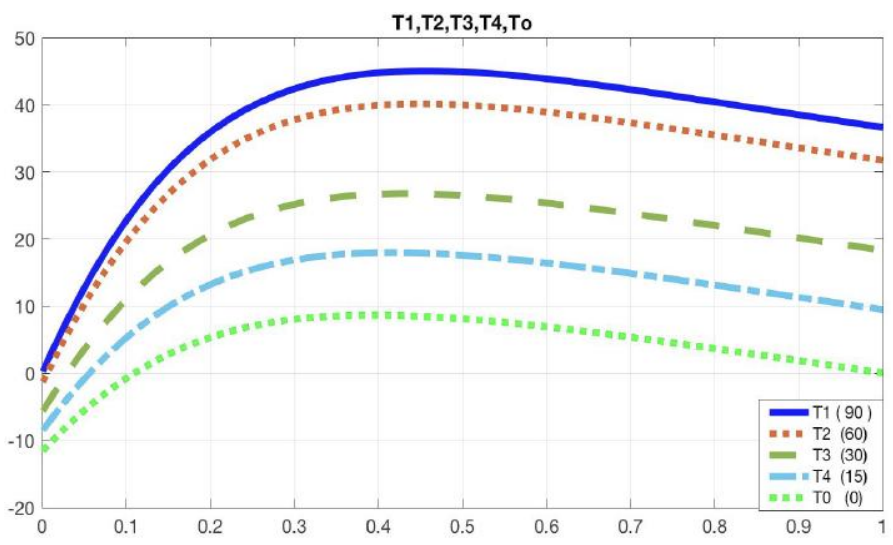

Figure 2. The control of the torque 


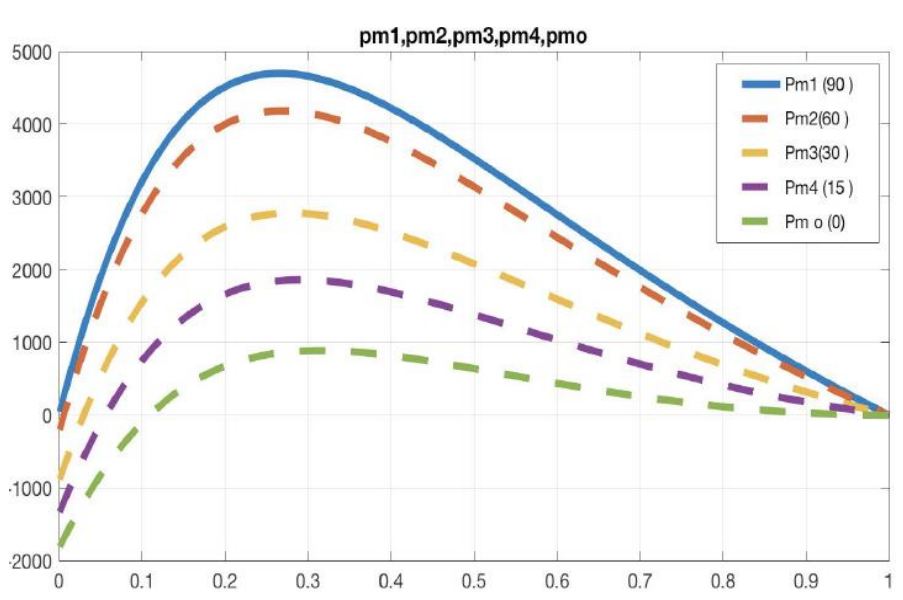

Figure 3. The control of Pm

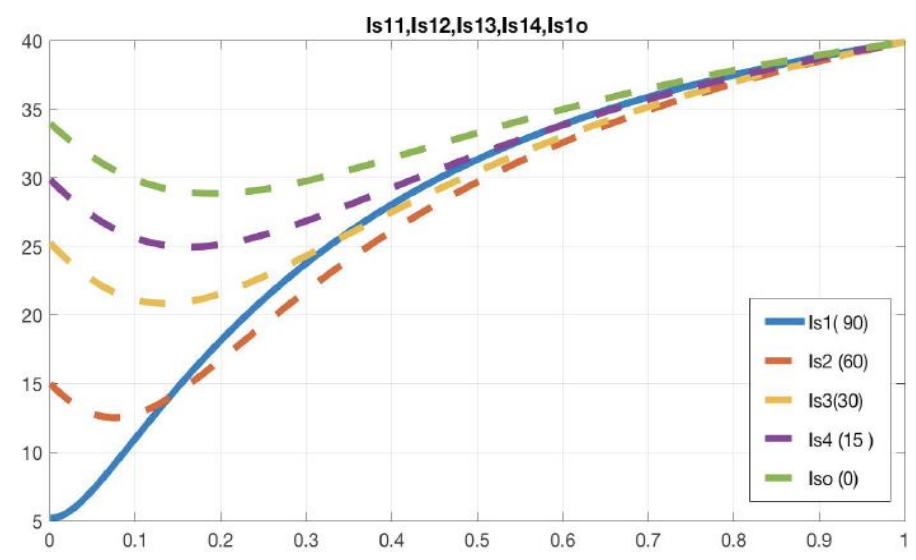

Figure 4. The control of ls1

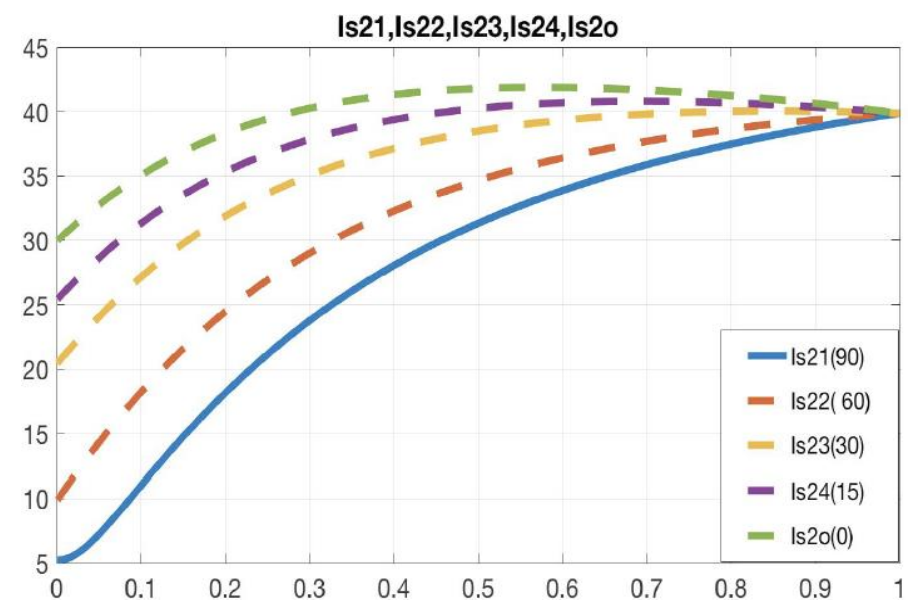

Figure 5. The control of 1s2

\section{RESULTS AND DISCUSSION}

The target achieved, as we derive a system equations of unbalanced two-phase induction motor could be applied for any running conditions of the motor, even it runs in balanced or un-balanced conditions according to the following equations: 


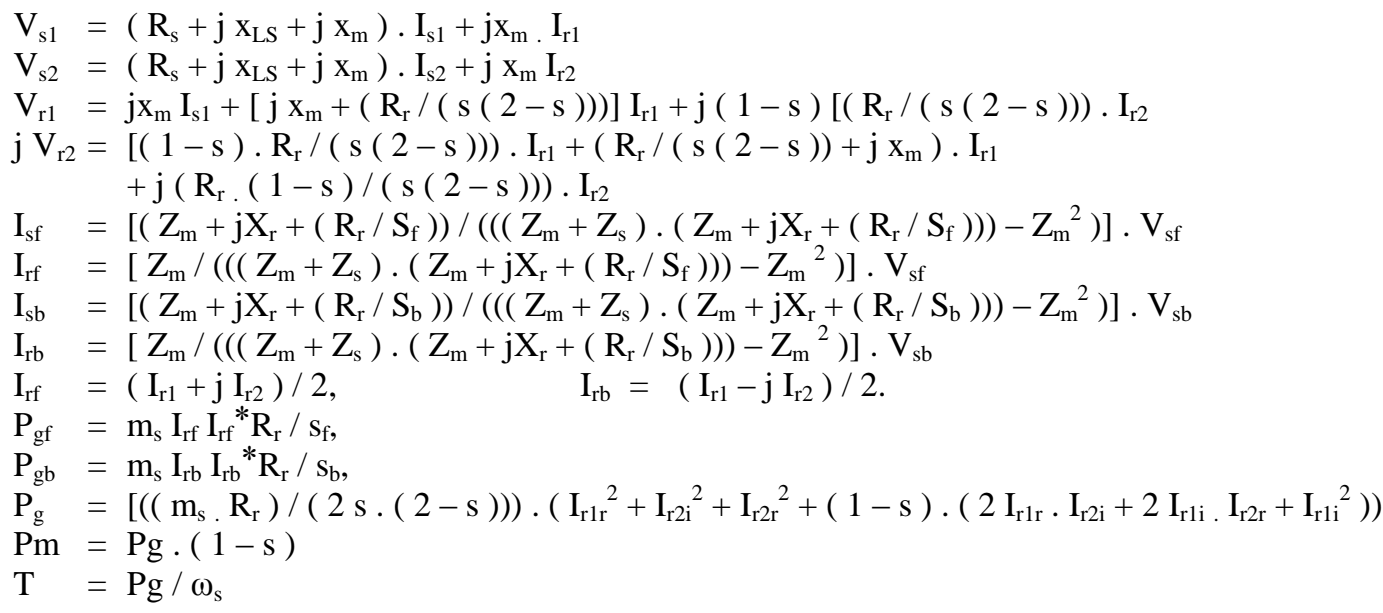

\section{CONCLUSION}

As there is no system driven especially for the two-phase induction motor fed from unbalanced two-phase supply, so we start for derivation the system equations for the said motor to be generally used even for the balanced or unbalanced two-phase supply. In this paper, we derive a system equation starting from the sequence equivalent circuit for the forward and backwards equivalent circuits, then we rearrange the equations with some mathematical assumptions which lead us to the new system equations. first for the voltage equations then for the current equations and finally for both power and torque equations. Moreover, we put an example which cover all cases with specific values and relations charts.

\section{REFERENCES}

[1] H. M. El Zoghby, S. M. Sharaf, and M. A. Ghazy, "Controlling of Two-Phase Servomotor by Changing The Phase Difference Angle Using Polynomial-Proportional Plus Integral (PPI) Controlle," Proceedings of the 14th International Middle East Power Systems Conference (MEPCON'10), 2010.

[2] M. Popescu, E. Demeter, D. Micu, V. Navrapescu and T. Jokinen, "Analysis of a voltage regulator for a two-phase induction motor drive," IEEE International Electric Machines and Drives Conference. IEMDC'99. Proceedings (Cat. No.99EX272), Seattle, WA, USA, 1999, pp. 658-660.

[3] H. S. Patel and R. G. Hoft, "Generalized Techniques of Harmonic Elimination and Voltage Control in Thyristor Inverters: Part I--Harmonic Elimination," in IEEE Transactions on Industry Applications, vol. IA-9, no. 3, pp. 310317, May 1973.

[4] V. D. Chikate, A. Y. Fadnis, G. C. Gondhalekar, "Speed Control of Two Phase Induction Motor," International Journal of Engineering Research in Electrical and Electronic Engineering (IJEREEE), vol. 4, no. 3, 2018.

[5] P. Záskalický, "Torque ripple calculation of two-phase im supplied by three-leg VSI inverter," Maszyny Elektryczne: zeszyty problemowe, 2017. 